\title{
Exact Polyhedral Machining
}

\author{
Cha-SooJun (csjun@,nongae.gsnu.ac.kr),Dong-Soo Kim \\ Dept. of IE and RECAPT, Gyeongsang National University, South Korea
}

Sehyung Park

Korea Institute of Science and Technology, South Korea

Key words: Polyhedral model, Topological information, NC tool path, Gouge

Abstract: Presented in this paper is a new approach to three-axis NC tool path generation for sculptured surfaces. In the proposed curve-based approach, the gouge-free tool paths are generated from a polyhedral model of STL form. The polyhedral model is offset using localoffsetting scheme. Then, the offset elements such as triangular facets, trimmed cylinders, and trimmed spheres are sliced by a series of drive planes. The curve segments on a drive plane are sorted, trimmed and linked, while the concave gouge is removed during the trimming process. The method is implemented on a PC, and some illustrative examples are provided in this paper. The main advantage of the proposed method is that a polyhedral model can be machined without any concave and convex gouge, especially on a NC machine supporting NURBS interpolation.

\section{INTRODUCTION}

One of the critical problems in generating tool-paths for a compound surface is cutter gouge (or interference). Various research works [1-7] have been done to solve the problem. There were several previous studies related to the gouge-free tool path generation for a polyhedral model, including those by Duncan [1], Choi [2], Hwang [3], etc. The polyhedralbased tool path generation approach is very robust in that it prevents the cutter from gouging the polyhedron without numerical stability problems.

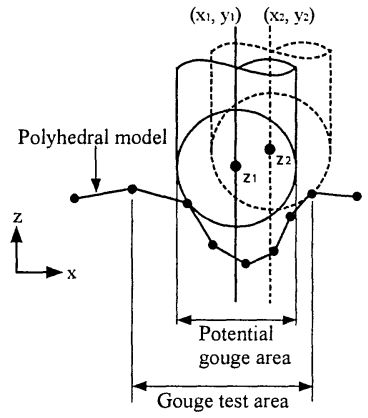

(a)

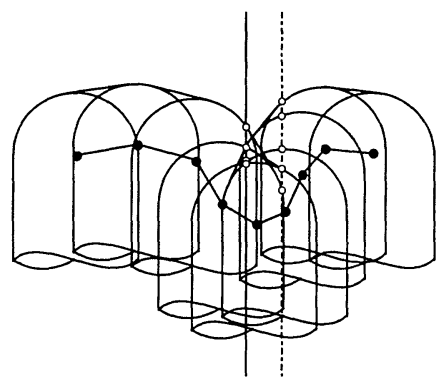

(b)

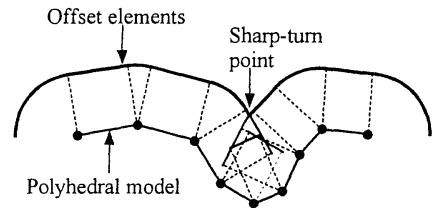

(c)

Figure 1. Point-based approaches and a curve-based approach to tool path generation for a polyhedral model (2D view)

The original version of this chapter was revised: The copyright line was incorrect. This has been corrected. The Erratum to this chapter is available at DOI: 10.1007/978-0-387-35392-0_40 
In the previous studies, for a given cutter center axis $\left(\mathrm{x}_{1}, \mathrm{y}_{1}\right)$, the highest cutter position $\mathrm{z}_{1}$ is determined by lowering the cutter until it touches any triangular facet under the cutter, as shown in Figure 1(a). Another method finds the intersection points of inverse tool offset surfaces with a cutter axis, and takes the highest point as the cutter position [5] (Figure 1(b)). The next position $\left(\mathrm{x}_{2}, \mathrm{y}_{2}, \mathrm{z}_{2}\right)$, which is distant by an adequate step-length from position $\left(\mathrm{x}_{1}, \mathrm{y}_{1}\right.$, $\left.\mathrm{z}_{1}\right)$, is calculated by the same process. The cutter moves from position $\left(\mathrm{x}_{1}, \mathrm{y}_{1}, \mathrm{z}_{1}\right)$ to position $\left(\mathrm{x}_{2}, \mathrm{y}_{2}, \mathrm{z}_{2}\right)$ linearly. Hereinafter, this method is called a 'point-based approach'. The pointbased approach has inherent limitations in the accuracy of convex gouge handling, and in identifying sharp-turn points in concave regions (Figure 1(c)). The sharp-turn points are used to tracing pencil-cut tool-paths [6].

A new method proposed in this paper is a 'curve-based approach'. In this approach, a tool path is generated as a compound curve, not as a sequence of points. Conceptually, this type of tool path can be obtained by intersecting the offset surface (or CL-surface [6]) with drive planes. However, it is very complex and time-consuming to calculate the offset surface of a polyhedron model exactly and completely. In this work, the offset elements of the model are calculated exactly in convex regions, but the ones in concave regions are overlapped. The overlapped portions are removed clearly after obtaining intersection curves. This method is simple and efficient, as well as can overcome the limitations of the point-based approach. The efficiency of the method comes from the fact that it makes better use of topological information and geometric characteristics of the polyhedral model, while most of the previous works treat the facets of the polyhedral model individually.

\section{OFFSET OF POLYHEDRAL MODEL}

Introduced in this section is a local-offsetting scheme for a polyhedral model. Since the global-interference between the offset elements that are not adjacent to each other, is complex and time-consuming to handle in a three-dimensional offsetting process, the scheme treats only the local-interference between adjacent offset elements in the process. Then, the globalinterference is removed during the two-dimensional CL-curve linking stage. Figure 2 depicts a $2 \mathrm{D}$ version of the local- and global-interference in the offsetting process. In Figure 2(b), the interference of the two offset facets is global in that the two offset facets are not adjacent to each other. In the offset model obtained by this scheme in Figure 1(c), the local-interference is cleared, but the global-interference (the overlapped portions in the convex region) remains. On the other hand, the offset model of Figure 1(b) has both local- and global- interference. Offsetting like Figure 1(b) may be called 'individual offsetting' comparing to the localoffsetting.

Based on the local-offsetting scheme, all the facets of the polyhedron are offset to the same triangular facet, translated to the normal direction of the facet by the amount of the cutter radius, and only the convex edges and vertices are offset to trimmed cylinders and trimmed spheres, respectively.

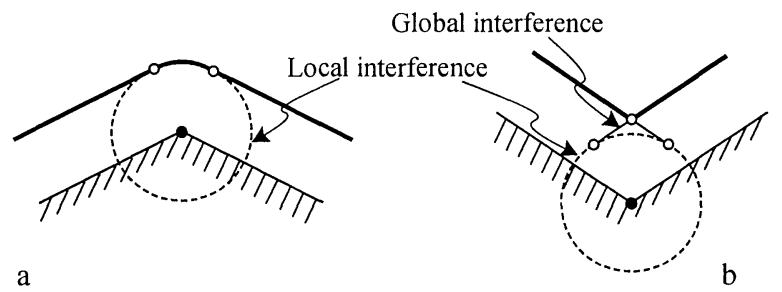

Figure 2. Local- and global-interference in offsetting (2D version) 


\subsection{Offset of an edge}

The attribute for the geometric characteristics of an edge can be classified into three different categories: convex, concave and flat. The attribute of an edge can be determined by the two adjacent triangular facets connected to the edge. As for an edge on the model boundary, in order to determine the attribute, a virtual facet parallel to the z-axis is adopted as shown in Figure 3.

Only a convex edge forms a cylindrical surface when it is offset (see Figure 4). The cylindrical face is bounded by four boundary curves: two circular arcs and two lines. The lines are coincident with the boundaries of the adjacent offset facets since the cylinder is trimmed by the facets. The circular arcs are represented in a quadratic rational Bezier curves.

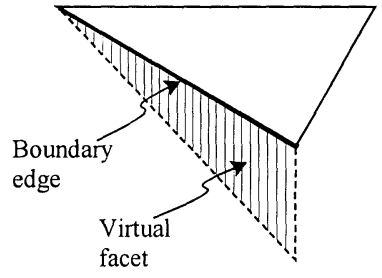

Figure 3. Determining the attribute of a boundary edge
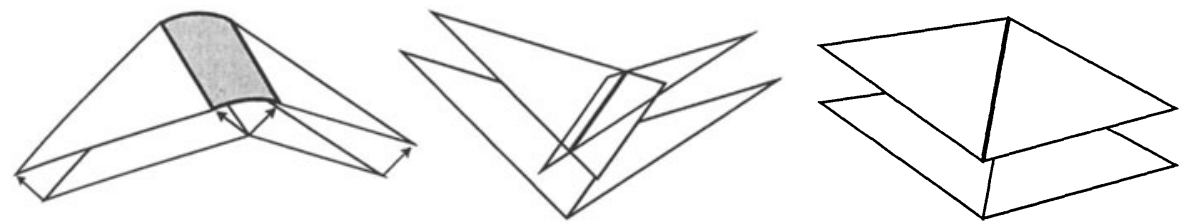

Figure 4. Offsetting of edges

\subsection{Offset of a vertex}

The attributes of geometric characteristics for a vertex are classified into convex, concave, flat, and saddle. Figure 5 shows the surfaces generated by offsetting different characteristic vertices. A spherical surface is generated from only the convex vertex. The spherical surface is bounded by circular arcs. The arcs are coincident with those of the adjacent cylindrical surfaces from the edges connected to the vertex, in that the sphere is trimmed by the cylinders. The offset surface is $\mathrm{G}^{1}$-continuous in the convex region.

The attribute of a vertex is defined by the attributes of the edges connected to the vertex. Table 1 shows the attribute of a vertex for various cases. To define the attribute of a vertex on

Table 1 Attribute of a vertex determined by the attribute of the attribute of connected edges

\begin{tabular}{|c|c|c|c|}
\hline $\begin{array}{c}\text { No. of } \\
\text { Edges }\end{array}$ & Attributes of the edges & Attribute of the vertex & $\begin{array}{c}\text { Spherical } \\
\text { surface }\end{array}$ \\
\hline \multirow{3}{*}{3} & All the edges are convex & Convex & Generated \\
\cline { 2 - 4 } & All the edges are concave & Concave & Not \\
\cline { 2 - 4 } & All the edges are flat & Flat & Not \\
\hline \multirow{2}{*}{4} & More than 3 edges are convex & Convex & Generated \\
\cline { 2 - 4 } & Otherwise & Saddle, concave, or flat & Not \\
\hline \multirow{2}{*}{$\begin{array}{c}\text { Over } \\
4\end{array}$} & $\begin{array}{c}\text { A new polyhedron with only convex } \\
\text { edges can be reconstructed }\end{array}$ & Convex & Generated \\
\cline { 2 - 4 } & Otherwise & Concave, saddle, or flat & Not \\
\hline
\end{tabular}




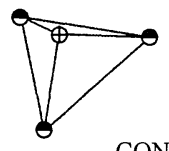

CONVEX

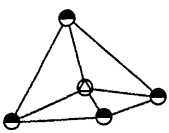

SADDLE
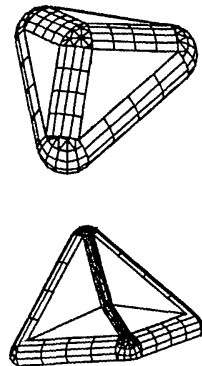

Figure 5. Offsetting of vertices with different attributes
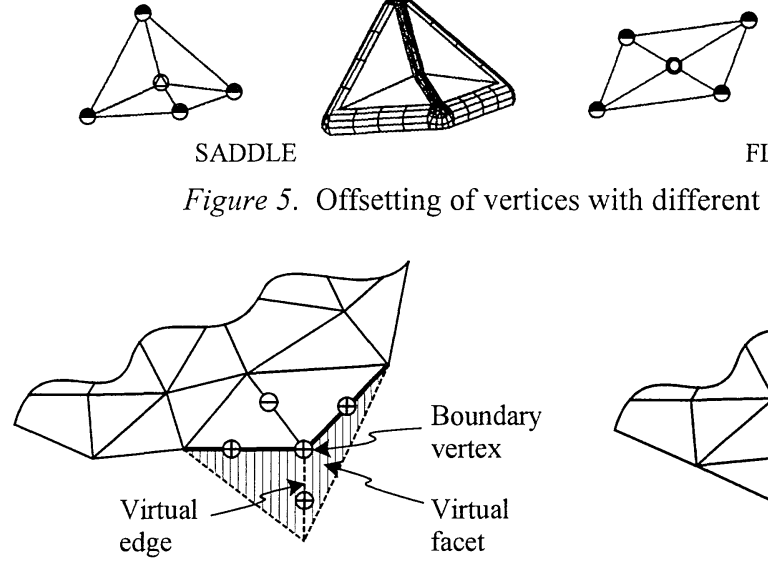

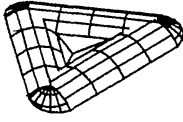

CONCAVE

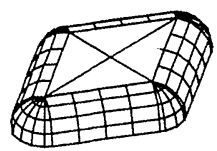

FLAT

Figure 6 . Defining the attribute of a boundary vertex

the model boundary, we adopt a virtual edge and two additional virtual facets that are vertical as shown in Figure 6.

When the attributes of the edges connected to a vertex are mixtures of convex, concave, and flat ones, it is not straightforward to define the attribute of the vertex. In this study, the polyhedron surrounding the vertex is reconstructed after removing the concave edges until all the edges become convex. If the number of the remaining convex edges is less then three, a spherical offset surface is not generated from the vertex. Figure 7 shows the procedure for reconstructing a polyhedron with convex edges to obtain the bounding curves of the offset spherical surface from the vertex. Note that the edge $\mathrm{E}_{1}$ is convex on the original polyhedron (Figure 7(a)), if its attribute changes to concave in the intermediate polyhedron (Figure 7(b)), and it is removed in the final model (Figure 7(c)). In the Figure 7(d), you can see that the cylinder $\mathrm{C}_{1}$, the offset element of the edge $\mathrm{E}_{1}$, is located outside of the trimmed boundary of the offset sphere. Therefore, the edge $E_{1}$ is of no use for generating the offset spherical surface. Figure 8 shows the boundary curves of the elements of the offset polyhedral model. As mentioned earlier, the offset elements at convex regions are exact, but the ones at concave regions are overlapped. The overlapped portions are removed in the CL-curve linking process.

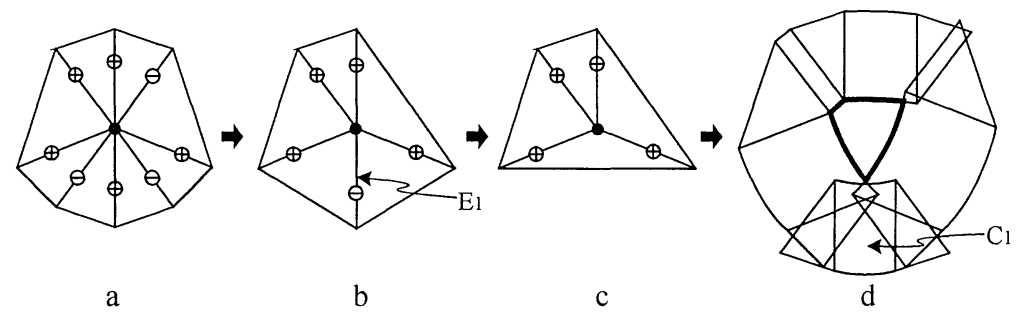

Figure 7. Procedure to obtain the bounding curve segment of the offset spherical surface from a vertex of a polyhedral model 


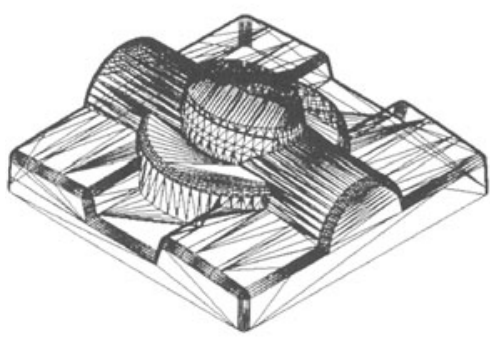

(a)

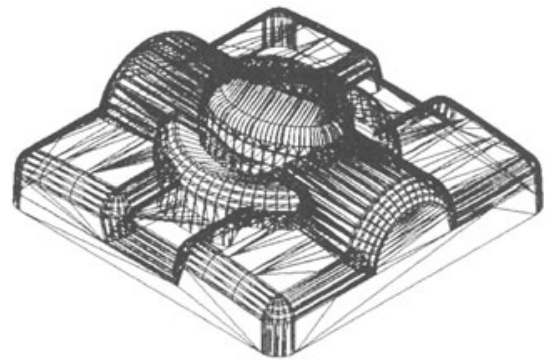

(b)

Figure 8. A polyhedral model and its local-offset model

\section{CL DATA GENERATION FROM POLYHEDRAL MODEL}

The overall procedure for generating tool paths is shown in Figure 9. The input data of the procedure are a polyhedral model of the STL form and the machining conditions, and the output is CL-paths of quadratic rational Bezier curve form.

\subsection{Slicing an offset triangle facet}

In the slicing process, instead of searching offset elements intersected with a given drive plane, a range of drive planes over an offset element is determined. Then, the element is sliced with the planes at once. The sliced curve segments are stored separately in the corresponding planes. This method reduces the time to search the offset elements and does not need to store the elements after slicing.

Since the drive planes are parallel to the xz-plane, the minimum and maximum $y$-values of a triangle are used to find the drive planes ranged over the triangle. The intersection between a plane and a triangle can be obtained simply by computing two intersection points between the plane and the edges of the triangle. The intersection line is called a cutterlocation curve (CL-curve).

\subsection{Slicing a cylindrical surface}

The offset of a convex edge forms a cylindrical surface, which is bounded by four curves: two circular arcs and two lines. The intersection between the cylindrical surface and a drive plane can be obtained by intersecting the bounding curves and the plane, and the result can be a straight line, an elliptic arc, or two elliptic arcs. The elliptic arc is represented by a quadratic rational Bezier curve(Figure 10).

\subsection{Slicing a spherical surface}

A convex vertex is offset to a spherical surface bounded by circular arcs. In order to obtain the intersection between a drive plane and a spherical surface, the intersecting points $\mathrm{V}_{0}$ and $\mathrm{V}_{2}$ between the plane and the bounding arcs of the surface are calculated first. Then, the center point $\mathrm{B}$ of the sphere is projected on the drive-plane to the normal direction of the plane to obtain the center point $C$ of the circle (Figure 11). The quadratic rational Bezier control point $\mathrm{V}_{1}$ and the weight at $\mathrm{V}_{1}$ are calculated by $\mathrm{V}_{0}, \mathrm{~V}_{2}$, and the center point $\mathrm{C}$. 


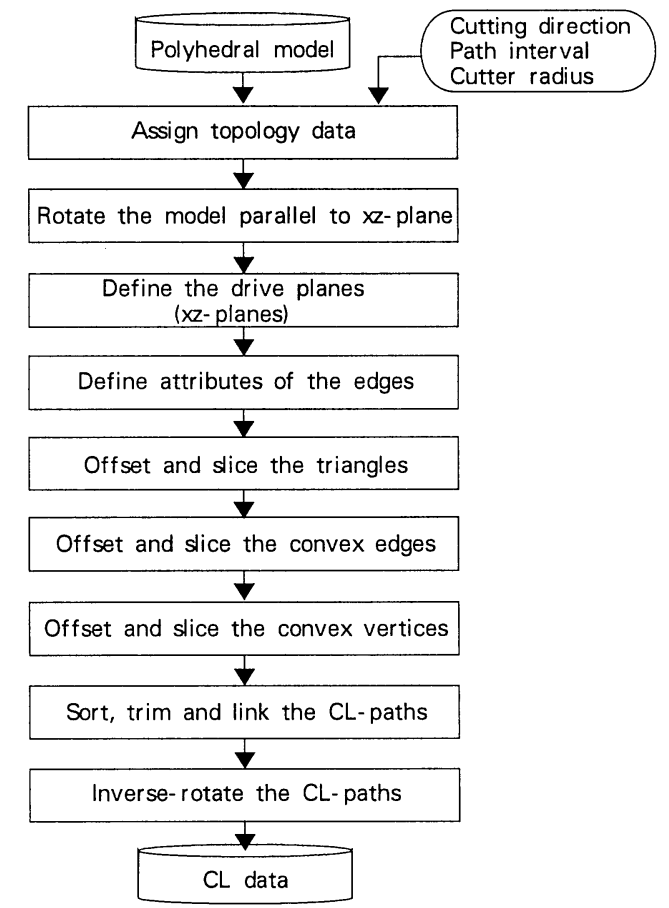

Figure 9. Overall procedure for $\mathrm{CL}$ data generation

\subsection{Sorting, trimming, and linking the CL-curves}

The CL-curves on a plane are sorted by the $x$-values of the start points of the curves. If two curves intersect, the curves are disjointed at the intersection points. The intersection point of line/elliptic-arc or line/line is computed analytically, while that of elliptic-arcs is obtained through a subdivision method. If two curve segments are overlapped, then the lower one is removed (see Figure 12). The resulting sequence of CL-curves becomes a CL-path, which is free of concave and convex gouge. Figure 13 shows the untrimmed CL-curves, and the CLpaths for machining a polyhedral model.

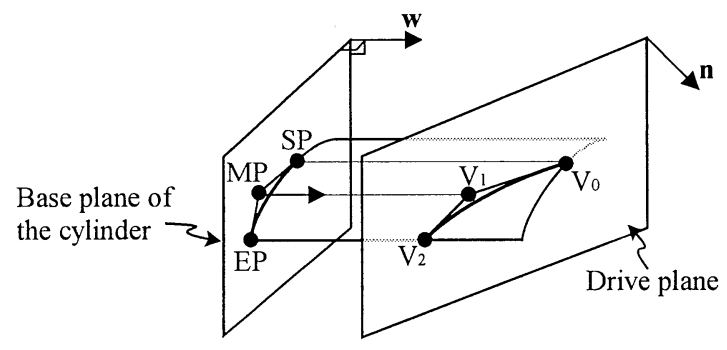

Figure 10. Intersection between a cylindrical surface and a drive plane 


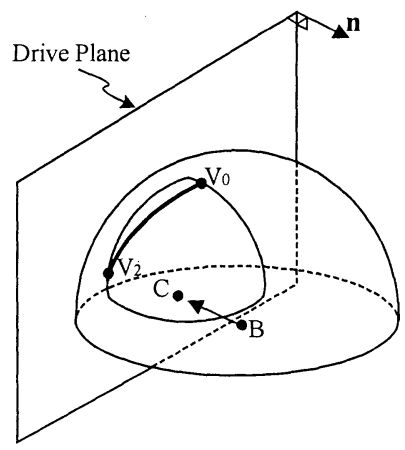

Figure 11. Intersection between a spherical surface and a drive plane

The CL-paths are directly converted to NURBS-type NC data for a machine supporting NURBS interpolation. As for a conventional NC machine, the elliptic arcs in the CL-paths should be approximated to line segments or circular arcs. In this approximation process, the tolerance control is much more robust than in the 'point-based approach'.

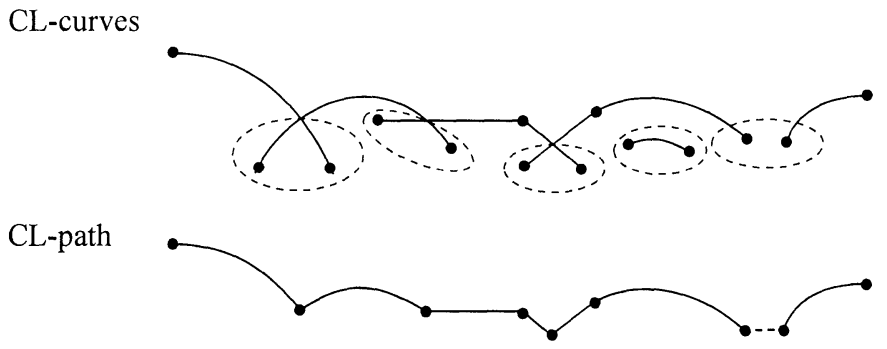

Figure 12. CL-curves and a CL-path

\section{ILLUSTRATIVE EXAMPLES AND AN EXTENSION OF THE METHOD}

In this study, the proposed method for generating gouge-free tool paths from a polyhedral model is implemented on a PC. Figure 14 shows the CL-paths obtained in the two cutting directions by the proposed method for machining a connecting rod. The polyhedral model has about 850 facets, and path-interval (or pick-feed) is $0.5 \mathrm{~mm}$. It takes under 5 seconds to generate the CL-data in Figure 14(b) on a $266 \mathrm{MHz}$ Pentium II machine.

Figure 15 shows another example of CL-paths, which is generated from the same polyhedral model as Figure 13. The CL-paths over the convex sharp-edge on the top face of the model prevents the sharp edge from being dulled by machine control errors. This example is provided to illustrate an extension of the proposed method. The tool paths are generated by slicing the Bezier control nets of the offset elements instead of the offset surfaces. Currently, the authors are working on the problem. 


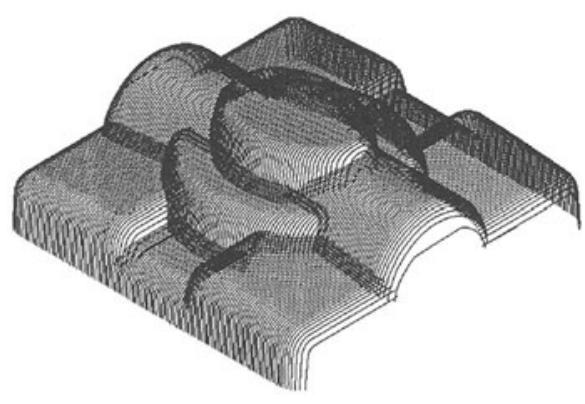

(a) untrimmed CL-curves

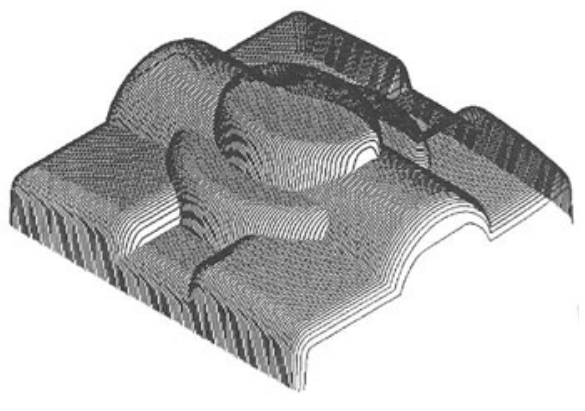

(b) CL-paths

Figure 13. Untrimmed CL-curves (a) and CL-paths (b)

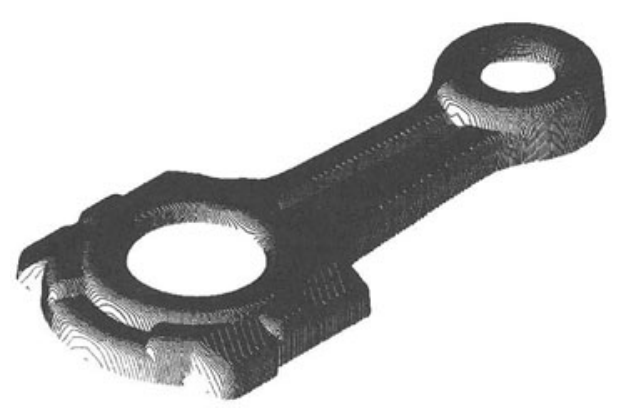

(a)

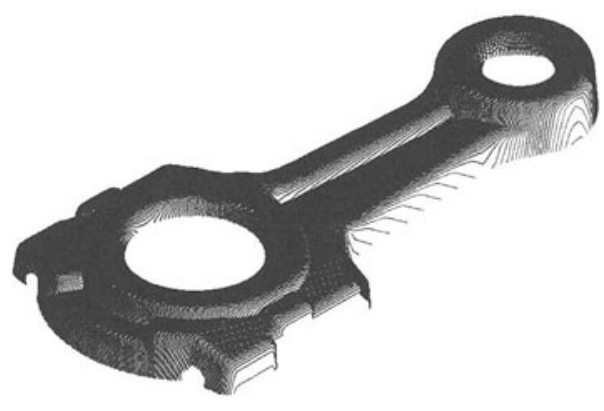

(b)

Figure 14 Examples of machining the connecting rod

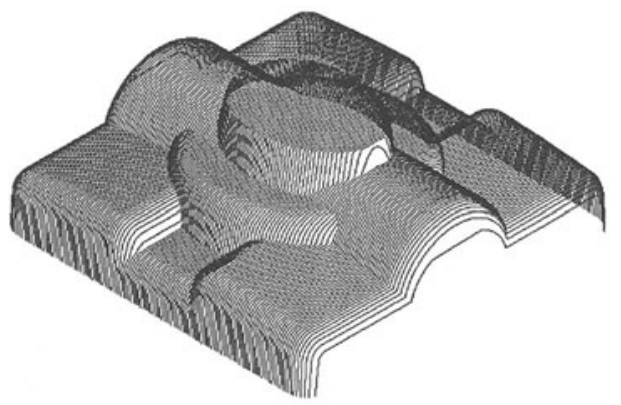

Figure 15. Convex sharp-edge handled CL-Paths for the model of figure 12 


\section{CONCLUDING REMARKS AND DISCUSSIONS}

A curve-based approach for generating gouge-free tool paths for three-axis machining of polyhedral models is proposed. The features of the approach are as follows.

(1) Robustness: The geometric elements treated in the algorithm are planes, cylindrical surfaces, spherical surfaces, lines, and elliptic arcs. Even the singularity cases can be handled without numerical stability problems. This feature is usually common for polyhedral-based tool path generation methods.

(2) Accuracy: Since the offset elements of the polyhedral model are not approximated and the intersections are computed exactly by analytical methods, the final CL data does not include approximation errors. A polyhedral model can be machined without any concave and convex gouge, especially on an NC machine supporting NURBS interpolation.

(3) Efficiency: The proposed method makes best use of topological information and the geometric characteristics of the edges and vertices (convex, concave, flat, and saddle) in order to check local-interference between offset elements. The global-interference, which is timeconsuming when checking in 3D-offsetting, is removed during $2 \mathrm{D}$ curve linking. In addition, instead of searching the offset elements intersecting a drive-plane, an offset element is sliced at once with a series of drive planes. This method reduces a lot of computation time.

With the proposed method, however, it is not easy to obtain contour-type tool paths. Further research will be conducted on handling convex sharp-edges, tool path generation for round-end mills, pencil-cut path tracing, and contour-type path generation.

\section{REFERENCES}

1. Duncan, J.P. and Mair, S.G., 1983, Sculptured Surfaces in Engineering and Medicine, Cambridge University Press, USA.

2. Choi, B.K. et al, 1988, 'Compound surface modeling and machining', Computer Aided Design, Vol.20, No.3, pp.127-136.

3. Choi, B.K. and Jun, C.S., 1989, "Ball-end cutter interference avoidance in NC machining of sculptured surface", Computer Aided Design, Vol.21, No.6, pp.371-378.

4. Hwang, J.S., 1992, 'Interference-free tool-path generation in the NC machining of parametric compound surfaces', Computer-Aided Design, Vol. 24, No. 12, pp. 667-676.

5. Takeuchi, Y. et al, 1989, 'Development of a personal CAD/CAM system for mold manufacturing', Annals of CIRP, Vol.38, No. 1, pp.429-432.

6. Choi, B.K., Kim, D.H. and Jerad, R.B., 1997, 'C-Space approach to tool-path generation for die and mold machining', Computer Aided Design, Vol.29, No.9, pp.657-669.

7. Lin, A.C. and Liu, H.T., 1998, 'Automatic generation of NC cutter path from massive data points', Computer Aided Design, Vol.30, No.1, pp.77-90. 